\title{
Areca Nut Disease Detection using Image Processing Technology
}

\author{
Dhanuja K C ${ }^{1}$, Mohan Kumar H P 2 \\ Department Of MCA \\ ${ }^{1}$ PES Engineering College Rd, PES College Campus, Mandya, Karnataka 571401
}

\begin{abstract}
The cultivation classification is one of the main steps in crop management. Classification may be used for various grades. The texture-based grading of the areca nut is suggested in this paper. Applications of Wavelet, Gabor, Local binary (LBP), Gray Level Difference Matrix (GLDM) and Gray Level Co-Occurrence Matrix (GLCM) are used to extract various texture features from areca nut. The Nearest Neighbor (NN) system is used. Experimentation with a dataset of 700 images from 7 classes to illustrate the efficiency of the proposed model. The Gabor wavelet features reflect 91.43 per cent of the classification limit.
\end{abstract}

Keywords-Precision agriculture, Crop analysis, Areca nut, Texture features, Nearest neighbor classifier

\section{INTRODUCTION}

Handling and meeting heightened pressures in agriculture from the environmental, economic, marketing and public sectors makes precision agriculture an effective concept for crop management[4]. Because of these stresses, farmer productivity and public benefits including ecological and environmental impacts are highly expected. To fulfill these standards the precision requirement agricultural production is highly recommended[5]. Agricultural automation can take advantage of machine vision capabilities, which can be used in various tasks such as inspection, grading, projected output, automated autonomous machine selection and guidance. Areca nut or Betel nut (Areca catechu L.,) is a crucial Indian commercial crop grown in various regions such as the East, the North East, the East and the West Ghats. India ranks first among other world countries according to both crop area and total production (47 percent). Indian areca nut production is in top rank with total world production (1.27 tones / ha)[5]. The grading of areca nut is an important stage in price fixing. This is helping farmers get the right price for their harvest. The research on the Areca classification is found in the To be divisive. An automated method for classifying Betel nut/Areca nut is proposed under [6]. The classification is achieved using the Areca nut 's color, shape and texture as characteristics. A method for classifying Areca nut in two classes was proposed based on color[2]. There are three steps in the method: segmentation, masking, and classification. The segmented color elements Red and Green Areca nut area is used for ranking. The use of texture characteristics for the classification of Areca nut has also been studied[3]. The first step is the segmentation of an isca nut from its history. Otsu processes conduct threshold segmentation on the global picture threshold. Mean gray level co-occurrence matrix (GLCM) and combination of these two characteristics are used for
Carried out using the classifier for Decision trees. An method for the grading of Areca nut has been proposed[1]. For segmentation the color characteristics with three sigma control limits are calculated. Areca 's Two grades Nut (boiling and non-boiling nuts)done using vector supporting machine (SVM) color tools. A survey is conducted for finding methods for the process of content-based image retrieval. In addition, the vector estimation of the function and various commonly used techniques are also evaluated[7].

\section{SCOPE AND OBJECTIVE}

We present a system where image analysis studies aiming at automated detection of disease that may be present in areca nut by using images of areca nut and bunches. In this work, we propose a convolution image processing model that has been trained with images of areca nut. The images contains areca nut of two region ,1 diseased arecanut, 2 non disease areca nut region .Evaluation of percentage of detection and HIS model shows that our proposed approaches will demonstrate stages of disease and accuracy if proposed methods

\section{PROPOSED SYSTEM}

The project's aim is to build a fully automated image classification system to positively identify different diseases in areca nut. In this project, we first perform disease detection on multiple areca nut, we used algorithms that provide good output at all detection stages. They have a comparatively higher predictability and a lower false positive rate.

\section{Advantages of proposed System}

- Faster execution of multiple images.

- Results can be obtained any time in a day

- Results can be obtained even without Expert agriculturist

- Detect the percentage of disease occurred

- Can scale to include bunch of areca nut

\section{SYSTEM ARCHITECTURE:}

Architecture Diagram is a graphical representation of the ideas that are part of architecture, their values, elements and components. These include system design diagrams for system engineers to grasp, explain, and convey ideas about the system configuration and user requirements that the system will meet. It's a basic framework that can be used in the planning phase of the system to help partners understand the architecture, discuss changes and clearly communicate intentions. 


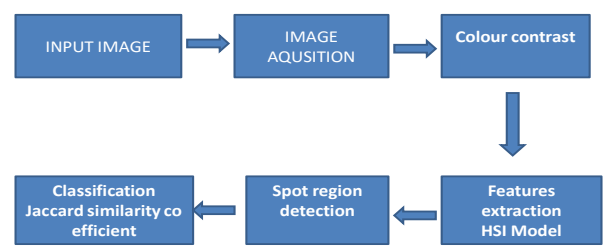

\section{Image acquisition system}

A computer vision program has been developed to take pictures of the areca nut. This unit involves a color camera with zooming lens and personal computer (the Intel Pentium $42.4 \mathrm{GHz}$ processer) (DFK-31AG03, Imaging Source, Inc.). The Opera CV 1.0, Intel Corporation (Opens Source Computer Vision Library) was related to 640/480 pixel RGB color graphics programs. The CCD camera was used for image acquisition with an aperture of $4600 \mathrm{~lx}$ and F4.0 (Iris diaphragm).

\section{Color contrast}

- A contrast expansion enhances the differences in brightness equally. The difference in shadows (dark), midtones (gray) or highlights (bright) regions increases across the dynamic spectrum of the picture while tonal improvement increases at the cost of differences in brightness in other regions.

- Gray-level histograms can be used to divide the total number of pixels to determine the percentage distribution of each gray level in the image by the number of times each gry-level value appears in the image.

- A linear transformation maps the lowest GLmin gray level of the image to zero and the highest GL max value in the picture to 255 is the simplest contrast stretch.

- The linear transformation for contrast enhancement uniformly spreads the gray-level values over the entire available contrast spectrum; hence, the relative shape of the histogram remains unchanged, but it expanded to fill the spectrum.

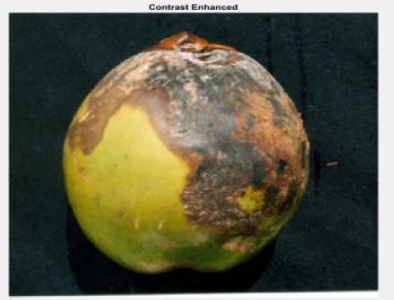

\section{Features extraction}

Firstly, it is important to define isca nut's principal axis. The centroid can be achieved as $\mathrm{X}=\mathrm{m}=1 \mathrm{xi} / \mathrm{m}$, and $\mathrm{Y}=1 \mathrm{yi} /$ $\mathrm{m}$, by assuming the binary image of the arc nut is $\mathrm{F}(\mathrm{xi}, \mathrm{yi})$ (where $\mathrm{I}=1,2, \mathrm{~m}$, and the pixel number is $\mathrm{m}), \mathrm{C}=($ alias $\mathrm{i}=1$
UiU T i)/m - MMT, where Ui is the ITH coordinate vector, and the mean vector is $\mathrm{M}=(\operatorname{locm} \mathrm{I}=1 \mathrm{Ui}) / \mathrm{m}$. $\mathrm{T}$ is the conversion of vectors. A pair of covariance matrix orthogonal eigenvectors is determined.

\section{HSI Model}

- HSI stands for Hue, Intensity and Saturation. Now when humans see an object of colour, its hue, saturation and brightness are identified.

- a special color (single yellow, orange or red) attribute.

- $\quad$ Saturation tests to what extent white light is diluting a pure color.

- Brightness is virtually impossible to measure; however, it depends on strength of light, which is a crucial factor in defining sensation of light. The strength is easy to calculate and the findings are also easy to interpret

- Hence the formula used to characterize an object in color is the HSI formula.

- The HSI models decouple light-carrying information in a color picture from the intensity (hue and saturation).

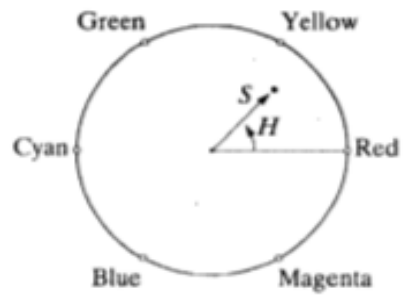

- The above model of HSI is displayed. The red axis angle gives the colour, the vector length is the saturation and intensity is determined by the position of the plane on the vertical axis.

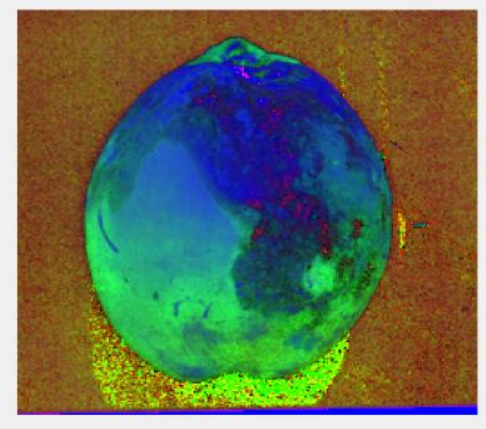

\section{Spot region detection}

Phase 1: Smooth operator transforms the actual arecanut image $\mathrm{p}(\mathrm{x}, \mathrm{y})$ into an image $\mathrm{f}(\mathrm{x}, \mathrm{y})$.

Phase 2: Use The gray level distribution on R, G and B belts shall be measured using the detection line (with scan resolution in pixels) moving on the main axis of an areca nut.

phase 3: Distinguish steady state (i.e., color, irisdiaphragm) between SR, HR and BR.

- if $\mathrm{fG}(\mathrm{x}, \mathrm{y})-\mathrm{fR}(\mathrm{x}, \mathrm{y})-<10 \mathrm{fR}(\mathrm{x}, \mathrm{y})-\mathrm{fG}(\mathrm{x}, \mathrm{y})$ $<150 \mathrm{fR}(\mathrm{x}, \mathrm{y}): \mathrm{fG}(\mathrm{x}, \mathrm{y}): \mathrm{fG}(\mathrm{x}, \mathrm{y}): \mathrm{fB}(\mathrm{x}, \mathrm{y})$, $\mathrm{fB}(\mathrm{x}, \mathrm{j})$; if $\mathrm{fG}(\mathrm{x}, \mathrm{y}), \mathrm{y})$ : 
- If $f G(x, y)>f R(x, y)>f B(x, y)$, then $f G(x$, $y)>f R(x, y)$, then $(x)$.

- If $\mathrm{fR}(\mathrm{x}, \mathrm{y})>180, \mathrm{fG}(\mathrm{x}, \mathrm{y})>180$ or $\mathrm{fR}(\mathrm{x}, \mathrm{y})$ $>\mathrm{fG}(\mathrm{x}, \mathrm{y}), \mathrm{fB}(\mathrm{x}), \mathrm{y})$, then $(\mathrm{x}, \mathrm{y})>\mathrm{fBR}$ where $f R(x i, y j), f G(x i, y j)$ or $f B(x i, y j)$ are pixel-coordinate functions of $\mathrm{R}, \mathrm{G}$ and $\mathrm{B}$.
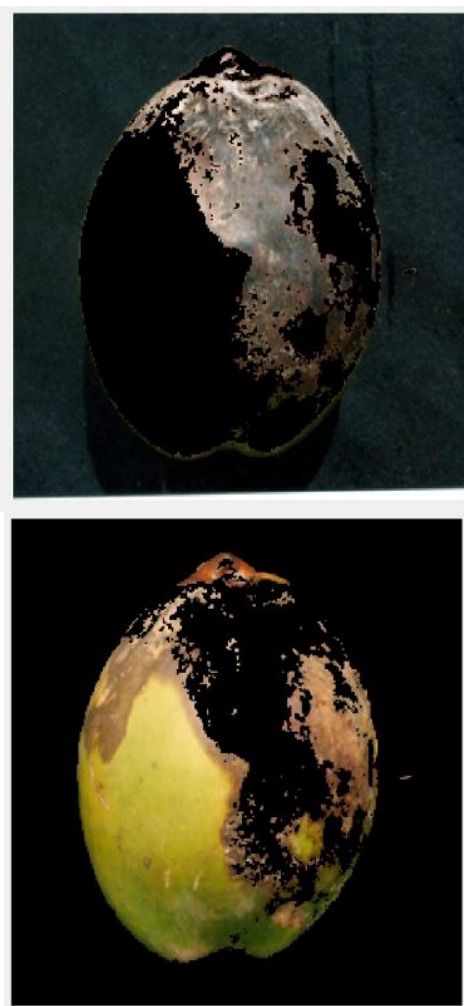

\section{Classification}

Geometrical and color characteristics have been used to determine the quality of the areca nuts. In order to define the areca nut consistency, three colors (i.e. mean gray level on the R-, G, and B bands: mean R, mean G, and mean B) were used to show 6 geometry features ( i.e. main axis lines, secondary axis, axis numbers, size, diameter, compactness). In any case where a pattern recognition system is available, the neural artificial (ANN) network has found numerous applications.

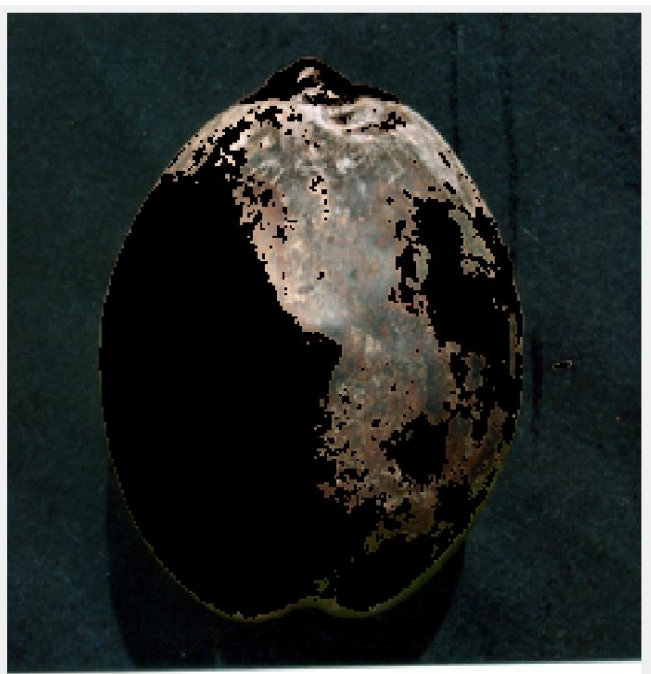

\section{Jaccard similarity co-efficient}

Jaccard similarity (Jaccard 1902, Jaccard 1912) is the standard index for binary variables. It is known as the quotient of variables compared to two objects between the intersection and paired union.

Equation

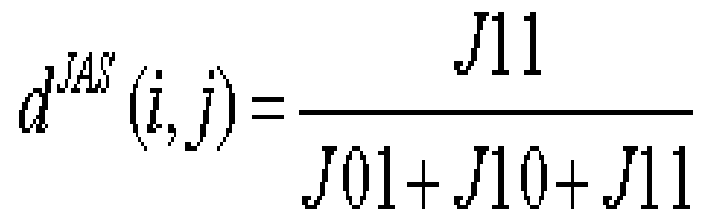

- In this dJAD equation the distance between the objects I and $\mathrm{j}$ is Jaccard. For two data records, the variable index $\mathrm{k}$ varies from 0 to $n-1$ with $n$ binary variables $y$. While comparing binary variables it is possible to distinguish four different combinations between $\mathrm{yi}, \mathrm{k}$ and $\mathrm{yj}, \mathrm{k}$. There are $(0 / 0),(0 / 1)$, $(1 / 0)$ and $(1 / 1)$ variations. You can group the sums of those combinations by:

- $\mathrm{J} 01$ : in $\mathrm{Yi}$, the total number is 0 and in $\mathrm{Yj}$ it is 1 .

- J10: full number of 1 in yi and 0 in yj.

- J11: total yi and yj variables are 1 ;

- J00: the total number of yi and yj variables is 0 .

Since-paired variable belongs to one of those classes it is easy to see:

$\mathrm{J} 00+\mathrm{J} 10+\mathrm{J} 11=\mathrm{n}$

J00 is discarded, as the similarity of Jaccard is based on a shared presence.

Dissimilarity to Jaccard is defined as dJAD $=1$ - dJAS.

For certain cases, the Jaccard-like value of $\mathrm{dJAS}=2 \mathrm{dBCD} /(1+\mathrm{dBCD})$ is calculated while $\mathrm{dBCD}$ is the Bray - Curtis difference. This equation does not contain binary values. Therefore, if a matrix is used on the one side and a matrix of count on the other, the results differ. If the count matrix is converted into a binary matrix already, the results are identical.

- Statistics used to gage sample sets similarity and diversity.

- Computs the intersection of the BW1 and BW2 binary images separated by the BW1 and BW2 unions, also known as the jaccard index.

- Separates the area affected from that of the undiseased zone.

- Computs an index of jaccard for each mark

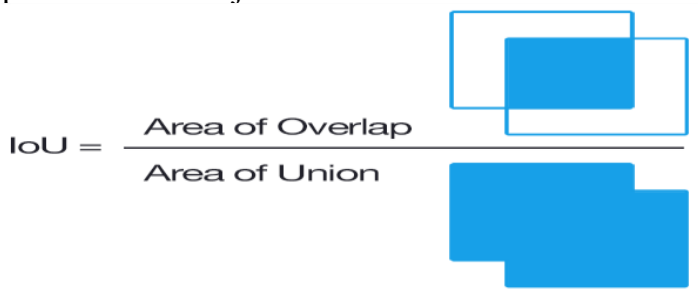




$$
\text { IoU: } 0.4034
$$

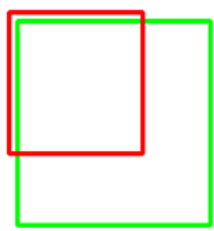

Poor
IoU: 0.7330

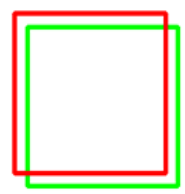

Good
IoU: 0.9264

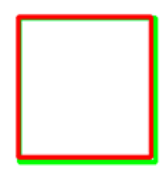

\section{Excellent}

Predict disease stage

Dice similarity coefficient

- It is a statistic used to gauge the similarity of two samples.

- Dice formula is intended to be applied to discrete data.

- This varies from the jaccard series, which lists only the positive once.

- DSC is a similarity quotient and ranges from 0 to 1

- $\quad$ DSC is used to measure predictive accuracy.

Where the cardinality of 2 sets is and

$$
\begin{array}{r}
\text { similarity }=2 *|X \cap Y| \\
-\cdots \\
-X|+| Y \mid
\end{array}
$$

The case sign represents the fixed intersection and represents the set number of components, $\mathrm{X}$ cardinality.

It measures the relation between sets $\mathrm{X}$ and $\mathrm{Y}$. The coefficient is 1.0. If both sets (i.e. contain the same elements), then $\mathrm{X}$ and $\mathrm{Y}$ have no similar elements, the coefficient is 0.0.0. If not, in it will be.

\section{RESULT AND DISCUSSION}

144 samples, including 49 Good, 46 Poor and 49 Negative, were sampled randomly in 287 frames, using a division of $50-5 \%$ in the BPNN classification. Eighteen hidden nodes have been gathered according to Eq. (1) 10 inputs, 3 outputs and 144 sample inputs. Using Mat laboratory functions 8.0, the BPNN classification was introduced. The set of samples was selected by random means for each setup until the BPNN converged. Fitting is also possible when there are any incorrect examples of BPNN in the training set. Because the amounts of ARACA nuts were already determined in training samples before the training process, it was doubtful whether What happened over fitting. The maximum number of iterations was set at 10,000 during the training cycle to guarantee a negligible effect of surge. More tests showed that when the convergence criterion for an error ratio was below 0.1 the same classification was achieved. The device was randomly checked with 48 Great, 46 Poor and 49 Bad pictures. The consistency of the classifications for Excellent Good and Bad grades is $91.7 \%, 89.1 \%$ and $91.8 \%$, respectively, and results are shown in Table 1. The accuracy on average was $90.9 \%$. The total number of correct and wrong classifications was 130 and 13. In this study the proposed algorithm is able to detect spots and identify the consistency of areca nuts with a CD camera accurately and effectively but it is not capable of inspecting the covered blades (or any other face). The automatic configuration of the system is made possible in the future for the classification of areca nuts.

\section{CONCLUSION}

In this study, the viewing system of the machine was developed to detect and identify the areca nodes as exceptional, decent and poor levels. The classification method was based on imaging techniques, DL algorithm and BPNN classification. Classification method The DL algorithm and the techniques of imaging allow for an efficient segmentation of defect regions. Three color characteristics (i.e. mean R, average $\mathrm{G}$, and average B) were achieved, six geometrical features (i.e. length of the principal axis, axis length, area and perimeter and compactness) and the spot areas. About 280 samples have been randomly split into training data sets and experimentation. With 144 training samples the BPNN classifier was created. The device was tested using the collection of test data. Test results show that with the established method, the areca nuts can be identified and graded efficiently.

\section{REFRENCES}

[1] Detection and classification of areca nuts with machine vision Kuo-Yi Huang 2012

[2] Classification of Diseased Areca nut based on Texture Features International Journal of Computer Applications (0975 - 8887) Recent Advances in Information Technology, 2014

[3] Segmentation and Classification of Raw Arecanuts Based on Three Sigma Control LimitsDecember 2012

[4] C.L. Hsieh, S.F. Cheng, T.T. Lin, Application of image texture analysis and neural network on the growth stage recognition for head cabbage seedlings, Journal of Agricultural Machinery 6 (2) (1997) 1-13 (in Chinese).

[5] D. Guyer, X. Yang, Use of genetic artificial neural networks and spectral imaging for defect detection on cherries, Computers and Electronics in Agriculture 29 (2000) 179-194.

[6] S.I. Cho, D.S. Lee, J.Y. Jeong, Weed-plant discrimination by machine vision and artificial neural network, Bio Systems Engineering 83 (3) (2002) 275-280. 